Mitteilungen der Deutschen Gesellschaft für Phlebologie

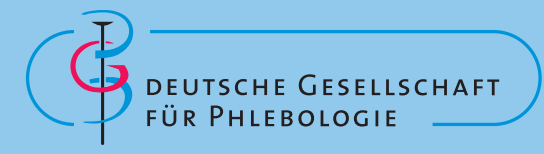

\section{AG Pflege}

Die AG Pflege ist fester Bestandteil unserer Gesellschaft. Mit hohem Engagement und Offenheit auch gegenüber den MFAs und Phlebologieassistentinnen übernimmt das Team in Hannover auch am Samstag am Vormittag eine Weiterbildungseinheit. Die Pflege, ambulant oder stationär, und die MFAs - sie sind ein wichtiger Bestandteil des Erfolgs, wenn wir Patienten behandeln.

Was wäre ein Arzt ohne sein Team? Was wäre er ohne die ambulanten Teams, die im Haus der Patienten als unser verlängerter Arm unsere Therapie umsetzen? Die DGP hat sich schon viele Jahre um die Einbindung dieser wichtigen Teamplayer gekümmert. Seit einigen Jahren findet freitags immer das Update Wundmanagement im Rahmen der DGP-Jahrestagung speziell für die Pflege statt. Seit 2014 findet unter Federführung von Dr. Renate Murena die curriculare Weiterbildung zum Phlebologieassistenten statt. Gleichzeitig eröffnete die DGP auch nichtärztlichen Mitgliedern die Möglichkeit, Mitglied in der DGP zu werden. In diesem Kontext wurde die AG Pflege gegründet. Wir waren damit weit vor unserer Zeit, denn erst seit Kurzem lässt z. B. die AWMF (Arbeitsgemeinschaft der wissenschaftlichen medizinischen Fachgesellschaften) nicht mehrheitlich ärztlich besetzte Gesellschaften überhaupt als Mitglieder zu.

Wie immer - der Anfang ist schwer. Nun sind wir aus den Kinderkrankheiten dieser Entwicklung herausgewachsen. Zum einen hat es sich etabliert, am Freitag das Update Wundmanagement als ICW-Rezertifiziertungssymposium mit 8 Punkten durchzuführen. Bei dieser Veranstaltung können ICW-zertifizierte Wundexperten, Pflegetherapeuten Wunde oder Fachtherapeuten Wunde die jährlich notwendigen Rezertifizierungspunkte erlangen, mit denen sie ihre Qualifikation in diesem Bereich erhal- ten können. Die Tagesveranstaltung ist natürlich auch offen für Ärzte und nicht ICWzertifizierte Pflegekräfte und MFAs.

Allerdings dreht es sich hier meist um das Ulcus cruris und alles, was damit in Zusammenhang steht: Diagnostik, Erscheinungsbilder, Differenzialdiagnosen, Therapieansätze, ganzheitliche Versorgung etc. Sehr wichtig, klar, aber nicht das Einzige, was wir im Team praktisch pflegerisch beherrschen oder diskutieren sollten. Frau Anja Kunz und Frau Birte Weiß, das engagierte Team, das der AG Pflege vorsteht, hat dankenswerterweise im Rahmen der DGPJahrestagung in Aachen ergänzend am Samstag einen Workshop zur Kompressionstherapie durchgeführt. Erstaunlich, wie viele ärztliche Kollegen noch nie BandageMaterialien persönlich in der Hand hatten oder gar selbst einen Strumpf oder Bandagen am Bein erfühlt haben! Der Workshop war ein kompletter Erfolg!

Anfang Dezember fand eine Telefonkonferenz zwischen der AG Pflege, dem Präsidenten der DGP (Prof. Stücker) und der Präsidentin des nächsten Kongresses (Dr.
Mendoza) statt. Wir alle waren hocherfreut über die sehr erfrischende Aufbruchstimmung. Zusätzlich zur bereits organisierten und zertifizierten ICW-Veranstaltung am Freitag, 30. September 2022, wird es in Hannover am Samstagmorgen ein zusätzliches Angebot für die Pflege, MFAs und Ärzte mit einem Schwerpunkt auf der Kompressionstherapie sowie der Diagnostik der arteriellen Verschlusskrankheit (AVK) geben. Wir wollen mit einem Theorie-Block beginnen und danach 2 Workshops (Kompression und AVK) anbieten. Wir möchten hiermit so eine Brücke zwischen AG Pflege, den Phlebologieassistenten und den Ärzten bauen. Ein reger, interdisziplinärer Austausch mit ausbaufähigen Ideen ist unser Ziel.

Gern möchte ich hier die Gelegenheit nutzen und das Team der AG Pflege kurz vorstellen ( $\triangleright$ Abb. 1). Die AG Pflege besteht derzeit aus 9 Mitgliedern aus der Pflege. Die unterschiedlichen Bereiche, in denen die Mitglieder beschäftigt sind (stationär, ambulant, Pflegeschule, Klinik), bereichern die Gruppe, und die unterschiedlichen Einblicke machen es möglich, ein breites Spek-
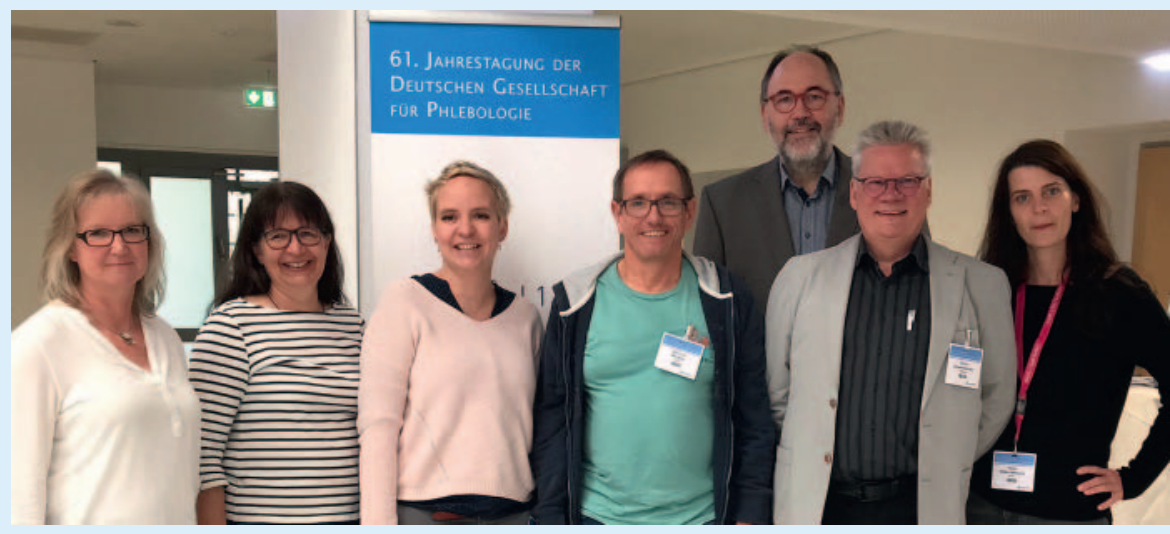

- Abb. 1 Mitglieder und Referenten der AG Pflege bei der der Deutschen Gesellschaft für Phlebologie in Münster. V.I. Birte Weiß, Anja Kuntz, Dr. Stine Lutze, Manfred Winkler, Carsten Hampel-Kalthoff, Bernd Assenheimer und Heike Hiller-Welsch. Quelle: Barbara Springer [rerif]. 
trum an Erfahrung einzubringen. Die Jahrestagung der DGP ist immer eine Gelegenheit, die Arbeit der Pflege im Rahmen des
Updates Wundmanagement vorzustellen und Fortbildungen mit ganz praktischen Ansätzen durchzuführen. Wir würden uns sehr über weitere Teilnehmer in der AG Pflege freuen, möchten hier auch speziell die MFAs ermuntern, mit dabei zu sein. 